\title{
A-WDL: An Improved Recommendation Algorithm for WDL based on Attentional Mechanism Adaptation
}

YuHao Xu ( $\nabla$ xyh0811@gmail.com)

Linyi University https://orcid.org/0000-0003-0334-2260

ZhenHai Wang

Linyi University https://orcid.org/0000-0002-3251-8862

Hui Chen

Linyi University

\section{Research Article}

Keywords: WDL, A-WDL, CTR prediction, Residual network, Attentional mechanism

Posted Date: September 9th, 2021

DOI: https://doi.org/10.21203/rs.3.rs-793351/v1

License: (c) (i) This work is licensed under a Creative Commons Attribution 4.0 International License.

Read Full License 


\title{
A-WDL: An improved recommendation algorithm for WDL based on attentional mechanism adaptation
}

\author{
Yu-Hao Xu ${ }^{1}$, Zhen-Hai Wang ${ }^{1}$, Hui Chen ${ }^{1}$
}

\begin{abstract}
CTR prediction is one of the main research directions of recommendation algorithm, which is widely used in e-commerce advertising recommendation and other fields. Wide\&Deep Learning (WDL) model is a CTR prediction and recommendation algorithm that combine linear model with deep neural network. To solve the problem that WDL is prone to over-fitting and the proportion of Wide and Deep learning part needs to be adjusted manually in practical application, Attentional WDL(A-WDL) algorithm is based on residual network to improve the Deep part. And use the attention mechanism to automate the learning of the Wide part and the Deep learning part of the proportion. Experiments on the public dataset Criteo on Kaggle show that the A-WDL algorithm improves the performance of AUC compared with other algorithms such as WDL, and effectively avoids the problem of overfitting. In addition, the performance improvement of A-WDL is explainable.
\end{abstract}

Keywords WDL· A-WDL. CTR prediction· Residual network· Attentional mechanism

\section{Introduction}

Due to the new round of technology and industrial revolution represented by new generation technologies such as cloud computing (Chen X, et al. 2019; Huang G, et al. 2019), Internet of Things (Chen X, et al. 2015; Chen X, et al. 2021) and mobile Internet [5], the way data is generated, acquired, and processed has been changed. It has enabled many fields to increasing intelligence and automation. it has especially promoted the development of e-commerce platforms, and also

\section{Zhen-Hai Wang} wangzhenhai@lyu.edu.cn

College of Information Science and Engineering Linyi University, Shandong Linyi, China promoted the generation and application of recommendation algorithms.

Among them, CTR prediction is part of the main research directions of recommendation algorithm, and is the core logic of bidding advertising. It widely uses in e-commerce advertising recommendation and other fields. In application scenarios such as online shopping platform, recommendation based on user personality is of major importance. For a product, the purpose of CTR prediction is tantamount to judging whether the user will click. By predicting CTR, the platform can more accurately grasp the user's preferences when pushing advertisements, which has a positive meaning for increasing order volume and user stickiness.

The currently popular CTR prediction methods include feature perception (Richardson M, et al .2007; Freudenthaler C, et al. 2010; Juan Y, Zhuang Y, Chin W 
S, et al 2016; Shan Y, et al 2016; Qu Y, et al 2016), serialized learning (Zhou G, et al 2019; Sun F, et al 2019), knowledge graph embedding (Wang H, et al 2018), etc. In addition, some methods, such as edge computing (Chen X, et al. 2019; Huang G, et al. 2017; Chen CM, et al. 2020), Reinforcement Learning (Chen X, et al. 2021), Computing Offloading (Lin B et al. 2020; Huang G, et al. 2017; Song H, et al. 2011), Automation Architecture (Chen X, et al. 2019; Chen X, et al. 2020; Huang G, et al. 2015; Liu X, et al. 2014; Huang G, et al. 2006; Huang G, et al. 2004), the successful application of Internet of Things (Huang G, et al. 2019) in other fields to promote the research of these methods in recommendation models (Zheng G, et al 2018; Gong Y, et al 2020; Song Q, Cheng D, et al 2020; Cui Z, et al 2020).

The A-WDL model is a feature-aware recommendation model that incorporates an automated architecture. The principle of the feature-aware recommendation model is to learn the relationship between features through a neural network to make recommendations. In 2007, the logistic regression model proposed by Richardson et al. (2007) was trying to use machine learning technology for CTR prediction of feature perception. The Logistic Regression model used Sigmoid activation function to map the results of linear regression to between $(0,1)$ and set a threshold value classifies the prediction results into two categories to predict whether the user will click on the advertisement, this processing was followed by subsequent models. In order to solve the problem that Logistic Regression model can only carry out firstorder interaction and is not strong in expression ability, Rendle et al. (2010) and Juan et al. (2016) proposed the
FM model and FFM model successively. FM model learns a latent weight vector for each feature vector on the basis of logistic regression. Then the implicit weight vectors are intersected pair to realize the learning of the nonlinear relationship between features. FFM model introduces the concept of field-aware on the basis of FM model, which further improves the expression ability of FM model, but at the same time leads to the increase of model complexity. FM and FFM models realize the learning of nonlinear relations, but due to the limitation of computing ability, only second-order feature interaction can be done in practical applications.

In 2016, the Deep Crossing model proposed by Ying et al. (2016) was trying to use MLP network for feature Crossing and achieved success. Qu et al. (2016) proposed the PNN model, proposed to use of product layers for feature cross, and proposed IPNN (Inner PNN) model and OPNN (Outer PNN) model according to the different product patterns. Cheng et al. (2016) proposed the WDL model, which combined machine learning model and Deep learning model. Logical regression model was used in the Wide part to capture low-order features, and MLP was used in the Deep part to conduct high-order feature interaction. After the output of the two parts are connected, the Sigmoid function is mapped to $(0,1)$ as the prediction result. WDL improves the prediction accuracy by combining machine learning model and deep learning model. In 2017, Wang et al. (2017) used Cross network to improve the Wide part of WDL and proposed the DCN model. Cross network used vector multiplication to Cross the features of input vectors to enhance the interaction between features. In the same year, 


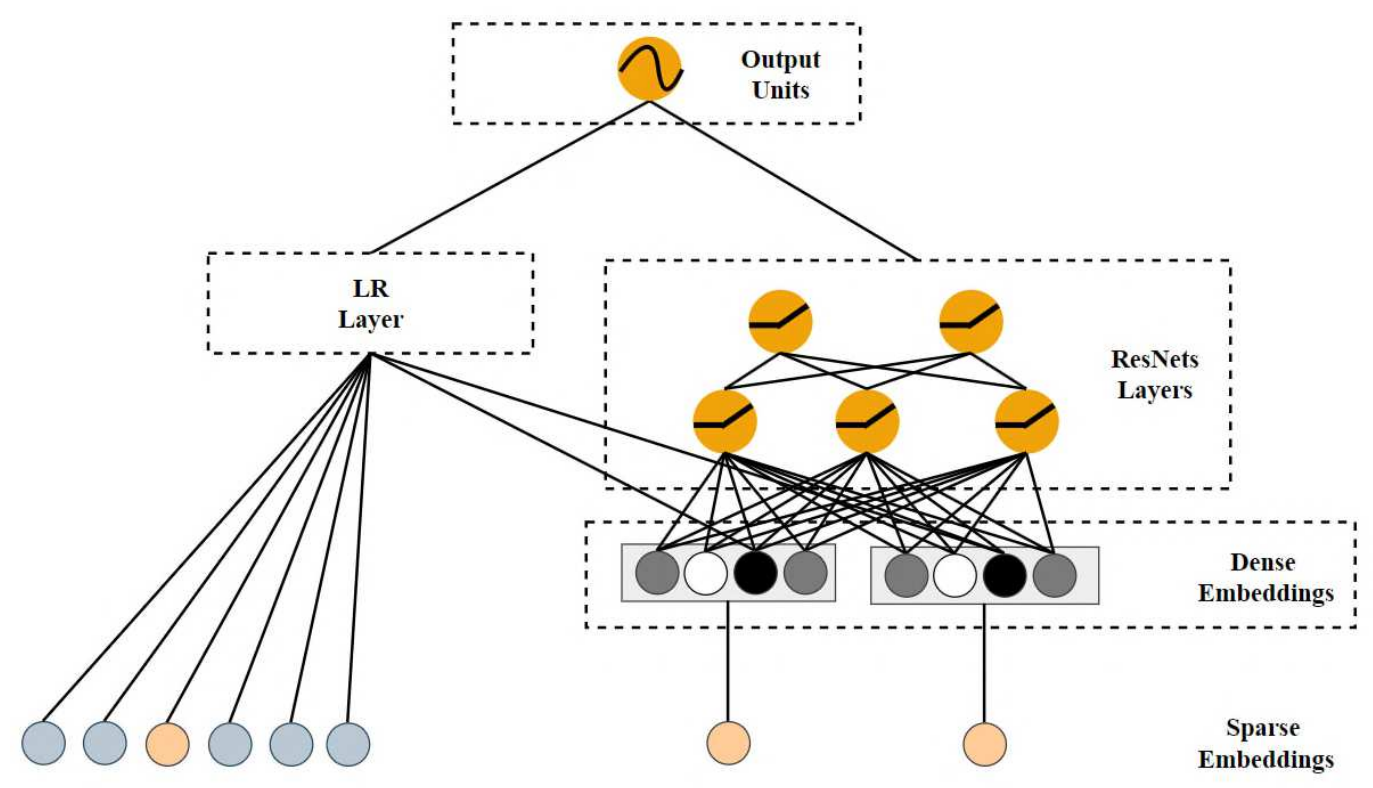

Fig. 1 The WDL models

Guo et al. (2017) used the FM model to improve the Wide part of WDL and proposed the DeepFM model, aiming to use FM to capture low-order feature interactions, which is the same improvement idea as DCN. In 2021, Wang et al. (2021) replaced the cross vector in the Cross network of DCN model with the cross matrix and proposed the DCN-M model, which mainly solves the problem of slow training under largescale data.

To sum up, the current CTR prediction models can be divided into three categories: machine learning model, deep learning model and hybrid model combining the two. Machine learning models include LR, FM, FFM, etc., which can be summarized as "memory-based recommendation". The original data often directly affects the recommendation results. Deep learning models include Deep Crossing, PNN, AFM, etc., which can be summarized as "generalization based recommendation". Through MLP automatic combination of features, potential feature combination methods can be mined. The hybrid model includes WDL, DCN, DEEPFM, DCN-M, etc., which can be summarized as "a recommendation that gives consideration to both basic capability and generalization capability". It is a combination of the advantages of machine learning model and deep learning model.

DCN, DeepFM, DCN-M and other models have achieved good results in improving WDL, but all the improvements made by these models are at the Wide part. These improvements to strengthen the "memory" ability of the model, but ignore the possibility of enhancing the "generalization" ability to improve the prediction effect. Meanwhile, the use of MLP on the Deep side of these models leads to overfitting during training. A-WDL model adopts the idea of improvement at the Deep part and improves MLP by using residual units (2016) to decrease the occurrence of overfitting during training. In addition, based on the characteristics of residual units, the problem of the disappearance of low-order feature interactions during forward propagation can be avoided when the number of network layers is high. Meanwhile, inspired by the application of automated architecture search CTR 
model (2020) in the field of CTR prediction, the AWDL model adds attention network to automatically adjust the proportion of Wide and Deep in the final prediction before summing the output of the two parts.

\section{Research Basis}

\subsection{WDL Model}

The WDL model is proposed based on the actual application scenarios of Google Play. By combining machine learning model and deep learning model, the WDL model realizes the interactive learning of loworder features and high-order features. Logical regression model is used at the Wide part to capture the strong correlation between a single feature and the target commodity, and the original data directly affects the recommendation results. The MLP model is used for advanced feature crossover in the Deep part to learn the nonlinear relationship between features, so as to improve the generalization ability of the model. The improvement of A-WDL model is based on WDL model.

\subsection{ResNet Unit}

ResNet was proposed by He et al. in order to solve the problem of over-fitting when the number of network layers in the image field is high. In 2016, Ying et al. applied the residual unit in the field of CTR prediction to reduce the over-fitting phenomenon prone to MLP, and confirmed the feasibility of using residual unit to improve the application of MLP in the field of CTR prediction.

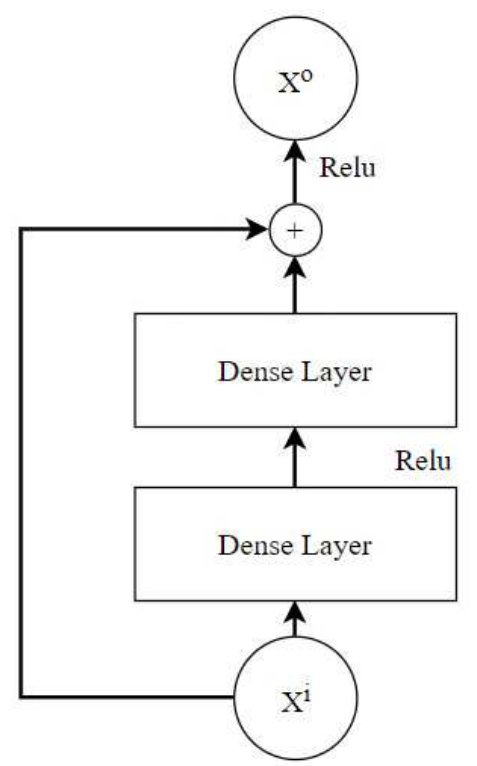

Fig. 2 The architecture of ResNet

The output $x^{o}$ of the residual element is calculated as follows:

$$
x^{o}=w_{2} \cdot\left(w_{1} \cdot x^{i}+b_{1}\right)+b_{2}+x^{i}
$$

Where $\mathrm{x}^{\mathrm{i}}$ is the original feature of the input, $w_{1}$ and $b_{1}$ are the weights and offsets of the first fully connected layer, $w_{2}$ and $b_{2}$ are the weights and offsets of the second fully connected layer. The AWDL model is improved by incorporating the idea of residual element in the Deep part. Residual units are used to improve MLP in A-WDL models to reduce overfitting. 


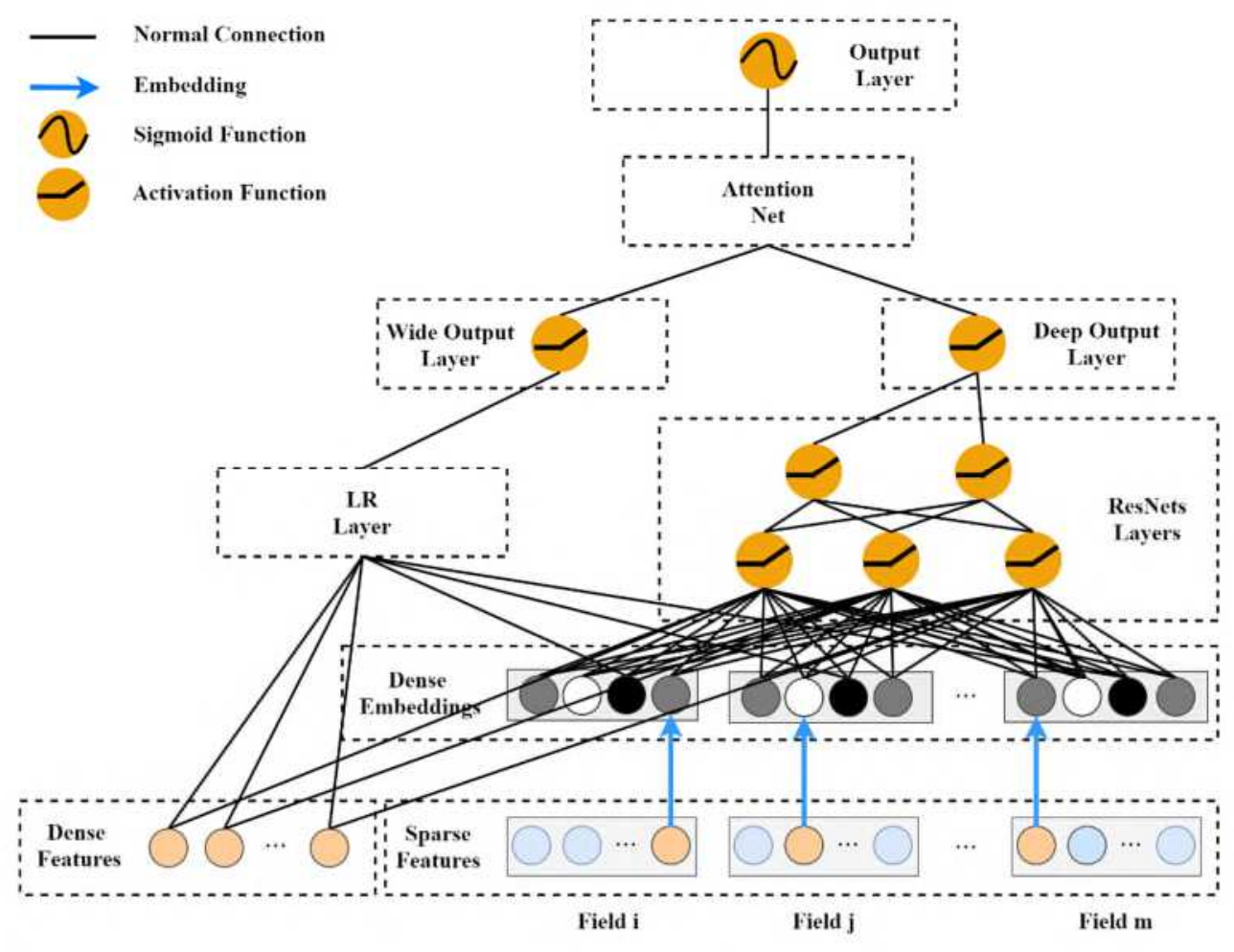

Fig. 3 The architecture of A-WDL

\subsection{Attention mechanism}

Introducing the attention mechanism into machine learning and deep learning models is usually to make the model selectively learn the input features, that is, to pay more attention to more important features.

In 2014, Volodymyr et al. (2014) applied the attention mechanism in the field of image and improved the effect of the model. In 2017, Xiao et al. (2017) combined the attention mechanism with the FMmodel and proposed the AFM model. AFM model increases the attention score for each cross term between the feature crossover layer and the output layer of FM, thus improving the effect of the FM model. In 2019, Huang et al. (2019) applied SENET (2018), which has achieved good results in the field of computer vision, to the recommendation algorithm and proposed a FIB-INET model using bilinear function learning feature interaction. In the same year, Zhou et al. (2019) proposed the DIEN model and introduced the attention mechanism on the basis of GRU to capture the relationship of temporal sequence characteristics. In 2020, Zhou et al. (2020) proposed an Interhat model that combines Transformer (2017) and recommendation algorithm to conduct feature interaction, which improves the efficiency of the algorithm and provides explainability for the importance of different feature interactions. The contribution of the attention mechanism in the final result of the Wide and Deep part of learning for automation in the A-WDL model. 


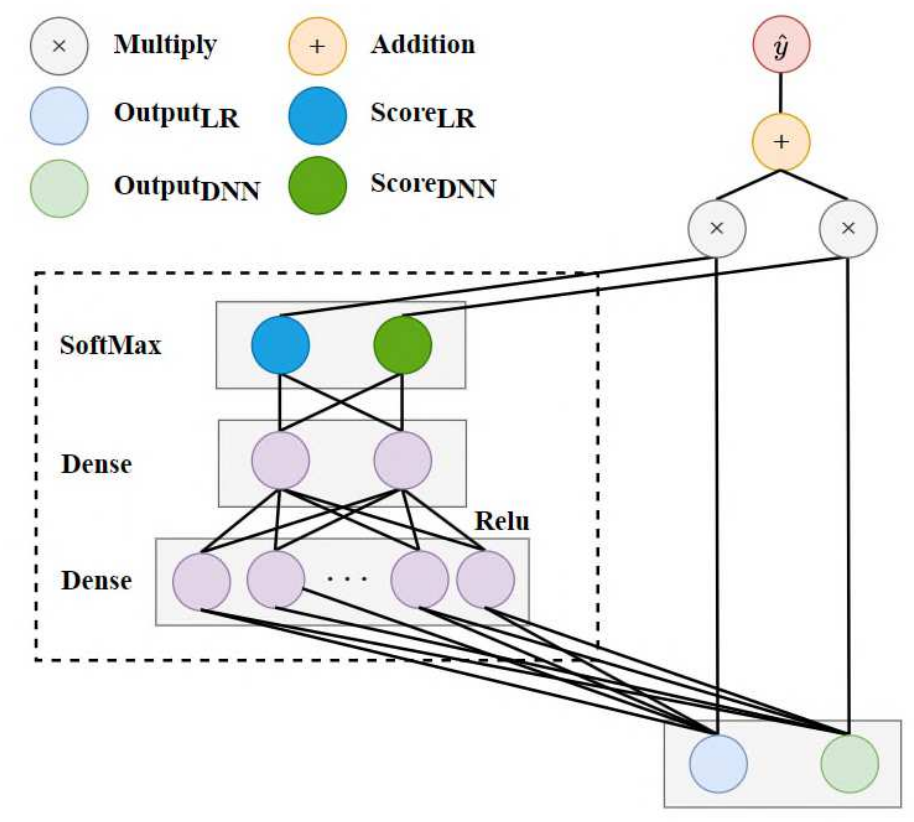

Fig. 4 The architecture of Attention Net

\section{$3 \quad$ A-WDL model structure}

The A-WDL model uses residual units to improve the MLP used by WDL at the Deep part, and based on the idea of attention mechanism, the proportion of the position automation learning in the final result of the combined output of the WDL model's Wide and Deep parts.

The input form of CTR prediction model is the sample of $(X, y)$, where $X$ is a set of eigenvalues, including a number of sparse features and dense features. The sparse features can be gender, educational background, etc., while the dense features can be age, salary, etc. $y \in\{0,1\}$ is the label corresponding to the sample, 0 means not clicked, 1 means clicked. The task of CTR prediction is to create a model and the output of that model:

$$
\hat{\mathrm{y}}=\text { CTR_model }(\mathrm{X})
$$

Where $\hat{y} \in(0,1)$ is used to predict whether the user will click on the current advertising.

\subsection{A-WDL model}

The goal of the A-WDL model is to improve on the Deep side, so no changes are made to the logistic regression model used in the Wide part; For the Deep part, the idea of residual units (2016) is introduced based on the MLP used by WDL for improvement. For the given original features, the dense features are normalized, and the sparse features are encoded as onehot and then processed by Embedding (2013).

Embedding is used to solve the problem of dimensional explosion and insufficient expression ability caused by one-hot encoding. It can reduce the dimensionality of high-dimensional data while maintaining the similarity between features after mapping to lower dimensions. In the recommendation system, Embedding is used to convert sparse features to dense features. After the feature processing is completed, input it to the Wide part and the Deep part for learning and get the output results. Multiply the results of the two parts with the attention score learned 
by the attention network to get the final output as follows:

$\hat{y}=\operatorname{Sigmoid}\left(\operatorname{Score}_{L R} \cdot y_{L R}+\operatorname{Score}_{D N N} \cdot y_{D N N}\right)(3)$

Where $\hat{y} \in(0,1)$ is the result of CTR prediction, usually by $\hat{y} \geq 0.5$ as the user may click on the item, and $\hat{y}<0.5$ as the user will not click to achieve the dichotomy; Score $_{L R}$ is the Attention Weight of the logistic regression part, and $y_{L R}$ is the output of the logistic regression part. Similarly, Score $_{D N N}$ is the Attention Weight of the DNN part, and $y_{D N N}$ is the output of the DNN part.

\subsection{Improvements in the Deep section}

Considering that the Deep Crossing model uses residual units to improve MLP effectively to prevent overfitting, the A-WDL model adopts a similar idea when improving the Deep part. A-WDL replaces the traditional MLP with multiple residual-error cells in tandem at the Deep part. The structure of the residual unit is shown in Figure 2, including two fully connected layers and a ReLU activated concatenation layer. The original input $\mathrm{x}^{\mathrm{i}}$ is spliced with the original input $x^{i}$ through a full connection layer that uses the ReLU activation function and a full connection layer that does not use the activation function. The output $x^{o}$ of the residual unit is obtained by activation through ReLU. Through the combination of multi-layer residual units, the high-order crossover of features is realized.

\subsection{Attention Net}

In A-WDL model, by introducing the idea of attention mechanism, the model can automatically learn and control the parameters of the proportion of Wide and Deep parts, namely, score LR $_{\text {and }}$ score $e_{D N N}$ in Formula (1), and adjust the contribution of Wide and Deep parts to the final result through these two parameters.

As showed in Figure 4, the input from the attention network is the output from the Wide and Deep parts of the A-WDL model, and the output is two attention scores score $_{L R}$ and score $_{D N N}$. The attention network consists of two fully connected layers and a SoftMax layer. The SoftMax layer uses the SoftMax function to compress the k-dimensional vector containing any real number into another $\mathrm{k}$ dimensional vector, such that the range of each element is between $(0,1)$ and the sum of all elements is 1 . The formula of SoftMax function is as following:

$$
P(y \mid x)=\frac{e^{h\left(x, y_{i}\right)}}{\sum_{j=1}^{n} e^{h\left(x, y_{i}\right)}}
$$

In the attention network, the SoftMax function is used to map the output value of the full connection layer to the $(0,1)$, and make their sum to be 1 . Then, the attention score is multiplied by $y_{L R}$ and $y_{D N N}$, which realizes the automatic learning of a weight value for Wide and Deep through the network. Making them makes different contributions to the final output.

\section{Experimental and analysis}

In this part, through the experimental comparison of A-WDL model and other models predicted by CTR, it is concluded that the effect of the A-WDL model is better. 

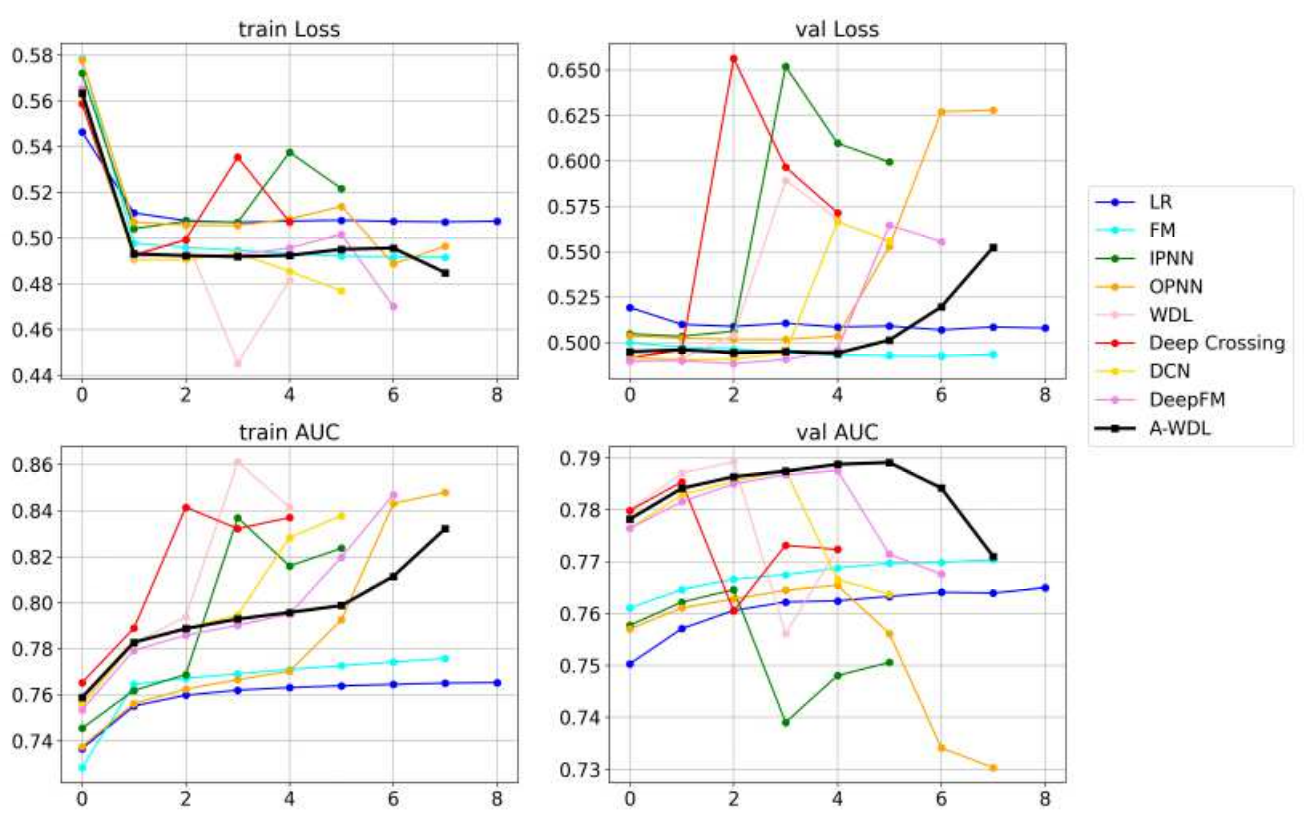

Fig. 5 The Loss curve and AUC curve on training set and validation set

\subsection{Dataset}

Use the public dataset Criteo on Kaggle. Criteo dataset is the click records of 45 million users after desensitization, including 13 continuous features and 26 classification features. The experiment uses the first 5 million data in the data set for testing, and randomly selects $80 \%$ of them as the training set. The remaining $20 \%$ are the test dataset.

\subsection{Evaluation Indicators}

Evaluation index of experimental use is a commonly used AUC (Area Under Curve) in dichotomous tasks. AUC was the size of the Area Under the ROC Curve, the value is between 0.5 and 1 . The closer the value is to 1 , the more authentic the detection method. AUC represents the probability of predicting that the score of the positive example is greater than that of the negative example when a positive example and a negative example are randomly taken out. AUC is used as an evaluation indicator because the number of positive examples in the data set in the field of CTR prediction is small, so the accuracy cannot measure the quality of the model. The purpose of CTR prediction application in industry is to recommend the items that are more likely to be clicked by users in the advertising goods, so AUC is usually used as an evaluation indicator.

\subsection{Comparative model}

The 7 most influential models in the field of CTR prediction: LR, FM, PNN, Deep Crossing, WDL, DCN, DEEPFM and A-WDL models were compared, in which PNN was further divided into IPNN and OPNN according to the different implementation methods of the product layer.

\subsection{Comparison between Loss and AUC}

By observing the loss and AUC index of each model in the training set and verification set in Figure 
5 , it can be found that, compared with other models, the loss value of the A-WDL model on the training set decreases steadily without significant fluctuations; 2) Compared with WDL, Deep Crossing, DCN and DEEPFM models, A-WDL model is less prone to overfitting.

Considering that the model will have overfitting problems in the verification set, the model enables the "early stop" strategy, which will stop iteration and save the optimal training results when overfitting occurred. Use the trained model to test on the test dataset to get the AUC results in Table 2, and use this table to compare the effects of the model. By observing Table 1, it can be found that: 1) On the test dataset, the AWDL model performs the best, and the effect is improved on the basis of the WDL model, which indicates that optimization of the network structure is effective in improving the effect of the model; 2) Compared with the improved DCN and DEEPFM models at the Wide part, the effect of the A-WDL model is also improved.

\section{Conclusion}

In this paper, a new model A-WDL is proposed, which is improved based on WDL model. The idea of residual unit is added in the Deep part, and the attention mechanism is added in the Wide and Deep part to realize automatic weight adjustment. This model can be utilized to CTR prediction. The experiment proves that it is feasible to improve the performance of the model for the Deep part. Compared with other models, AWDL model has the following advantages: 1) It is not easy to appear overfitting phenomenon in the training process; 2) Adaptively adjust the contribution of the
Wide and Deep parts to the final result through the attention mechanism. Experiments on real data set (Criteo data set) show that the A-WDL model 1 has no obvious overfitting on both the training set and the test dataset; 2) The performance of AUC in the test dataset was better than that of other models.

Tab. 1 AUC comparison with other models

\begin{tabular}{|c|c|}
\hline Model & Test Dataset AUC \\
\hline LR & 0.7642 \\
\hline FM & 0.7703 \\
\hline IPNN & 0.7620 \\
\hline OPNN & 0.7653 \\
\hline Deep Crossing & 0.7813 \\
\hline WDL & $\mathbf{0 . 7 8 0 3}$ \\
\hline DCN & 0.7846 \\
\hline DeepFM & $\mathbf{0 . 7 8 6 2}$ \\
\hline A-WDL & $\mathbf{0 . 7 8 9 9}$ \\
\hline
\end{tabular}

Future work directions include 1) using FM or FFM improvements at the Wide part, and considering integrating the attention mechanism into the FM or FFM model; 2) use other ideas of newer attention mechanisms to improve attention networks.

\section{Conflicts of interest}

Ethical approval: Not applicable.

Funding: This study was funded by National Natural Science Foundation of China,

Project No.: 61901206.

Conflict of interest: The authors have no conflicts of interest to declare that are relevant to the content of this article.

Informed Consent: Not applicable.

Authors' contributions: The main ideas and model design of the paper were realized by Yuhao $\mathrm{Xu}$ and Zhenhai Wang. The data collection and experiment 
were completed by Yuhao $\mathrm{Xu}$ and Hui Chen. The experiment was guided by Zhenhai Wang. The first draft of the manuscript was written by Yuhao $\mathrm{Xu}$, and all authors commented on previous version of the manuscript. All authors read and approved the final manuscript.

\section{References}

Chen X, Lin J, Ma Y, Lin B, Wang H, Huang G (2019) Selfadaptive Resource Allocation for Cloud-based Software Services Based on Progressive QoS Prediction Model. SCIENCE CHINA Information Sciences, 2019, 62(11):1-3. Huang G, Luo C, Wu K, Ma Y, Zhang Y, Liu X (2019) SoftwareDefined Infrastructure for Decentralized Data Lifecycle Governance: Principled Design and Open Challenges. IEEE International Conference on Distributed Computing Systems. Chen X, Li A, Zeng X, Guo W, Huang G (2015) Runtime modelbased approach to IoT application development. Frontiers of Computer Science, 2015, 9(4):540-553.

Chen X, Li M, Zhong H, Ma Y, Hsu C (2021) DNNOff: Offloading DNN-based Intelligent IoT Applications in Mobile Edge Computing. IEEE Transactions on Industrial Informatics. https://doi.org/10.1109/TII.2021.3075464.

Huang G, Liu X, Ma Y, Lu X, Zhang Y, Xiong Y (2019) Programming Situational Mobile Web Applications with Cloud-Mobile Convergence: An Internetware-Oriented Approach. IEEE Transactions on Services Computing, 12(1): 6-19.

Richardson M, Dominowska E, Ragno R (2007) Predicting Clicks: Estimating the Click-Through Rate for New Ads. WWW2007: The 16th International World Wide Web Conference:521-530.

Freudenthaler C, Schmidtthieme L, Rendle S(2010) Factorization machines factorized polynomial regression models. ICDM
2010: The 10th IEEE International Conference on Data Mining:1-6.

Juan Y, Zhuang Y, Chin W S, et al (2016). Field-aware Factorization Machines for CTR Prediction. RecSys '16: The 10th ACM Conference on Recommender Systems.

Shan Y, Hoens T R, J Jiao, et al (2016). Deep Crossing: WebScale Modeling without Manually Crafted Combinatorial Features. KDD '16: The 22nd ACM SIGKDD International Conference on Knowledge Discovery and Data Mining.

Qu Y, Han C, Kan R, et al (2016). Product-Based Neural Networks for User Response Prediction. ICDM: 2016 IEEE 16th International Conference on Data Mining:1149-1154.

Zhou G, Mou N, Fan Y, et al (2019). Deep Interest Evolution Network for Click-Through Rate Prediction. AAAI 2019: The Thirty-Third AAAI Conference on Artificial Intelligence.

Sun F, Liu J, Wu J, et al (2019). BERT4Rec: Sequential recommendation with bidirectional encoder representations from transformer. ACM2019: Proceedings of the 28th ACM international conference on information and knowledge management:1441-1450.

Wang H, Zhang F, Xie X, et al (2018). DKN: Deep knowledgeaware network for news recommendation. Proceedings of the 2018 world wide web conference:1835-1844.

Chen X, Chen S, Ma Y, Liu B, Zhang Y, Huang G (2019). An Adaptive Offloading Framework for Android Applications in Mobile Edge Computing. SCIENCE CHINA Information Sciences.

Huang G, Xu M, Lin X, Liu Y, Ma Y, Pushp S, Liu X (2017) ShuffleDog: Characterizing and Adapting User-Perceived Latency of Android Apps. IEEE Transactions on Mobile Computing:2913-2926.

Chen CM, Huang Y, Wang KH, Kumari S, Wu M (2020) A secure authenticated and key exchange scheme for fog computing. Enterprise Information Systems, 1-16. 
Chen X, Zhu F, Chen Z, Min G, Zheng X, Rong C (2021) Resource Allocation for Cloud-based Software Services Using Prediction-Enabled Feedback Control with Reinforcement Learning. IEEE Transactions on Cloud Computing. https://doi.org/10.1109/TCC.2020.2992537.

Lin B, Huang Y, Zhang J, Hu J, Chen X, Li J (2020) Cost-Driven Offloading for DNN-based Applications over Cloud, Edge and End Devices. IEEE Transactions on Industrial Informatics. 16(8): 5456-5466

Huang G, Xu M, Lin X, Liu Y, Ma Y, Pushp S, Liu X (2017) ShuffleDog: Characterizing and Adapting User-Perceived Latency of Android Apps. IEEE Transactions on Mobile Computing. 16(10): 2913-2926.

Song H, Huang G, Chauvel F, Xiong Y, Hu Z, Sun Y, Mei H (2011) Supporting runtime software architecture: A bidirectionaltransformation-based approach. Journal of Systems and Software, 84(5): 711-723.

Chen X, Lin J, Ma Y, Lin B, Wang H, Huang G (2019) Selfadaptive Resource Allocation for Cloud-based Software Services Based on Progressive QoS Prediction Model. SCIENCE CHINA Information Sciences. (11): 219101

Chen X, Wang H, Ma Y, Zheng X, Guo L (2020) Self-adaptive Resource Allocation for Cloud-based Software Services Based on Iterative QoS Prediction Model. Future Generation Computer Systems:287-296.

Huang G, Ma Y, Liu X, Luo Y, Lu X, Blake M (2015) ModelBased Automated Navigation and Composition of Complex Service Mashups. IEEE Transactions on Services Computing. 8(3): 494-506.

Liu X, Huang G, Zhao Q, Mei H, Blake M (2014) iMashup: a mashup-based framework for service composition. Science China Information Sciences. 54(1): 1-20.
Huang G, Mei H, Yang F (2006) Runtime recovery and manipulation of software architecture of component-based systems. Automated Software Engineering. 13(2): 257-281.

Huang G, Liu T, Mei H, Zheng Z, Liu Z, Fan G (2004) Towards Autonomic Computing Middleware via Reflection. International Computer Software and Applications Conference.

Huang G, Liu X, Ma Y, Lu X, Zhang Y, Xiong Y (2019) Programming Situational Mobile Web Applications with Cloud-Mobile Convergence: An Internetware-Oriented Approach. IEEE Transactions on Services Computing. 12(1): 6-19.

Zheng G, Zhang F, Zheng Z, et al (2018) DRN: A deep reinforcement learning framework for news recommendation. WWW2018: Proceedings of the 2018 World Wide Web Conference:167-176.

Gong Y, Jiang Z, Feng Y, et al (2020) EdgeRec: recommender system on edge in Mobile Taobao. Proceedings of the 29th ACM International Conference on Information \& Knowledge Management. 2020:2477-2484.

Song Q, Cheng D, Zhou H, et al (2020) Towards automated neural interaction discovery for click-through rate prediction. The 26th ACM SIGKDD International Conference on Knowledge Discovery \& Data Mining:945-955.

Cui Z, Xu X, Xue F, et al (2020) Personalized Recommendation System Based on Collaborative Filtering for IoT Scenarios IEEE Transactions on Services Computing:685-695

Heng-Tze C, Levent K, Jeremiah H, et al (2016) Wide \& Deep learning for recommender systems. DLRS2016: The 1st Workshop on Deep Learning for Recommender Systems.

Ruoxi W, Bin F, Gang F, et al (2017) Deep \& Cross Network for Ad Click Predictions. KDD 2017: The 23th ACM SIGKDD Conference on Knowledge Discovery and Data Mining. 
Guo H, Tang R, Ye,Y, et al (2017) DeepFM: A FactorizationMachine based Neural Network for CTR Prediction. The International Joint Conference on Artificial Intelligence.

Wang R, Shivanna R, Cheng D, et al. DCN V2: Improved Deep \& Cross Network and Practical Lessons for Web-scale Learning to Rank Systems[C]. //WWW2021: The Web Conference 2021: International World Wide Web Conference. 2021.

He K, Zhang X, Ren S, et al (2016) Deep Residual Learning for Image Recognition. CVPR2016: The 29th IEEE Conference on Computer Vision and Pattern Recognition:770-778.

Mnih V, Heess N, Graves A, et al (2014) Recurrent Models of Visual Attention. NIPS'14: The 27th International Conference on Neural Information Processing Systems:2204-2212

Xiao J, Ye H, He X, et al (2017) Attentional Factorization Machines: Learning the Weight of Feature Interactions via Attention Networks. IJCAI 2017: The 26th International Joint Conference on Artificial Intelligence.

Huang T, Zhang Z, Zhang J (2019) FiBiNET: combining feature importance and bilinear feature interaction for click-through rate prediction. RecSys '19: The 13th ACM Conference on Recommender Systems:169-177.

Hu J, Shen L, Sun G (2018) Squeeze-and-Excitation Networks. CVPR2018: Proceedings of the IEEE Conference on Computer Vision and Pattern Recognition:7132-7141.

Li Z, Cheng W, Chen Y, et al (2020) Interpretable Click-Through Rate Prediction through Hierarchical Attention. WSDM '20: The 13th International Conference on Web Search and Data Mining:313-321

Vaswani A, Shazeer N, Parmar N, et al (2017) Attention Is All You Need. Advances in Neural Information Processing Systems 29.
Mikolov T, Chen K, Corrado G, et al (2013) Efficient Estimation of Word Representations in Vector Space. ICLR2013: The 1st International Conference on Learning Representations. 